\title{
El cambio didáctico y sus consecuencias en el profesorado de matemáticas y ciencias
}

\author{
Miriam MÉndez CoCA \\ Centro Universitario Villanueva de Madrid \\ mmendez@villanueva.edu \\ David MÉnDEZ CoCA \\ Centro Universitario Villanueva de Madrid \\ dmendez@villanueva.edu
}

\begin{abstract}
Resumen
Para conseguir resultados más óptimos en los procesos de enseñanza-aprendizaje de las matemáticas y ciencias en la actualidad se propone la mejora de la formación de los profesores. Esta preparación tiene tres dimensiones: formación inicial de grado donde se adquieran los conocimientos necesarios, una formación pedagógica para además de tener el conocimiento saberlo transmitir y una formación permanente en metodologías y actualización de contenidos para poder responder de la forma más eficaz a las necesidades y demandas de esta sociedad globalizada del conocimiento.
\end{abstract}

Palabras clave: Metodología; formación permanente; enseñanza matemática;cooperativo

\section{The didactic change and consequences in training of math and science teachers.}

\begin{abstract}
In order to get better marks in mathematics and science it is proposed to improve the training of the math and science teachers. The training has three dimensions: the Bachelor degree is acquired with the learning of the contents of these subjects, pedagogical learning to teach the contents in a better way and a lifelong learning didactic methods and update contents to response to the demands of this knowledge global society.
\end{abstract}

Key Words: Methodology; lifelong learning; cooperative; math learning

\section{Referencia normalizada:}

Méndez Coca, M.; Méndez Coca, D. (2013) El cambio didáctico y sus consecuencias en el profesorado de matemáticas y ciencias. Historia y Comunicación Social. Vol. 18 N$^{\circ}$ Especial Noviembre. Págs. $29-40$.

Sumario: 1. Introducción 2. El profesor en su papel docente 2.1. La formación inicial del profesorado: conocimientos y habilidades profesionales 2.2. La formación metodológica del profesor 2.2.1. Cuestiones previas al aprendizaje cooperativo2.2.2. Variables del aprendizaje cooperativo2.2.3. Modalidades del aprendizaje cooperativo2.2.4. El aprendizaje cooperativo en matemáticas2.2.5. Decisiones y actividades del profesor en el aprendizaje cooperativo3. Conclusiones 4. Bibliografía 


\section{Introducción}

Vestigios de argumentación para hacer cambios en el ámbito educativo, en especial en ciencias y matemáticas, se multiplican en la actualidad. La atención al cambio, como procedimiento de progreso en la educación para el conocimientose recogeen los documentos que la Unión Europea produce en la actualidad: el Informe Cifras Clave de la Educación en Europa 2012, (Eurydice, 2012a) ofrece una combinación de datos estadísticos e información cualitativa sobre las principales dimensiones de la educación en la UE, constituyendo una destacada aportación al debate sobre la política educativa en el espacio europeo. Se afirma taxativamente en el Prólogo, la necesidad de avanzar en la formación para el progreso de la educación europea (Vassiliou y Semeta, 2012: 3). Con similar orientación, en el Informe sobre La Enseñanza de las Matemáticas en Europa. Retos comunes y políticas nacionales, hallamos algunas de las razones exhibidas con mayor frecuencia respecto de los sucesivos cambios que afectan al sistema educativo y formativo de profesores de matemáticas y de sus alumnos en las actuales circunstancias, partiendo de la información aportada por los datos estadísticos de Eurostat y de los estudios PISA y TIMSS que, a nivel internacional, se están llevando a cabo sobre la enseñanza de las matemáticas, las ciencias y la lengua. Las mencionadas evaluaciones se elaboran siguiendo un marco conceptual y metodológico común, precisamente para ofrecer indicadores que pudieran ser de utilidad para las nuevas políticas educativas a formular por parte de los Estados y cambiar objetivos, contenidos y métodos del proceso de enseñanza-aprendizaje matemático. "El indicador que recibe más atención a nivel público es la posición relativa de cada país según la puntuación media que obtiene en las pruebas. Desde la década de los sesenta, la puntuación de cada país se ha convertido en un factor que afecta considerablemente a las políticas educativas nacionales y que genera presiones para adoptar las prácticas educativas de aquellos países con un rendimiento más elevado" (Eurydice, 2012b:15).

En este caso, la temática se centrará en la formación inicial y permanente del profesorado para la aplicación de las metodologías cooperativas en el escenario de los procesos educativos.

\section{El profesor en su papel docente}

En el informe sobre "La Enseñanza de las Matemáticas en Europa", se explicitan tres aspectos en la formación del profesor: los conocimientos sobre la materia a impartir, las competencias y habilidades para enseñarlos y la comprensión de los procedimientos mediante los cuales aprenden mejor los alumnos. La enseñanza eficaz de las matemáticas depende en gran medida de la destreza de los profesores. Por tanto, sus conocimientos sobre la materia - los principios y procesos matemáticos - y su formación resultan cruciales. Sin embargo, una buena enseñanza no solo depende de los conocimientos y habilidades matemáticas del profesor, sino también de su 
comprensión sobre cómo enseñar la materia y sobre cómo aprenden los alumnos y ambos aspectos son esenciales para que el profesor reflexione sobre las necesidades de sus alumnos y les dé una respuesta adecuada (Eurydice, 2012b). Del texto se infiere que la formación del profesor comprende los conocimientos específicos, las reglas pedagógicas y las destrezas sobre los procedimientos didácticos adecuados al aprendizaje de los alumnos respecto de la materia de que se trate. En consonancia con este planteamiento se afirma en el informe que "existe un acuerdo generalizado sobre la correlación entre la calidad de la enseñanza y la formación del profesorado, por una parte, y el rendimiento de los alumnos, por otra, incluido el rendimiento en matemáticas (...) La UE ha reconocido hace tiempo esta conexión y considera la formación del profesorado y el apoyo que presta a los docentes como un elemento muy importante dentro de los sistemas educativos europeos" (Eurydice, 2012b: 127).

\subsection{La formación inicial del profesorado: conocimientos y habilidades profesionales}

En la práctica totalidad de los países de la UE los candidatos a profesores titulados deben poseer estudios académicos de las materias de que se trate y de los componentes profesionales que les habilitan para la docencia en el nivel educativo correspondiente.

a) Modelos básicos de formación inicial. En la UE dos son los modelos de formación inicial del profesorado que imparten enseñanzas regladas: modelo simultáneo y consecutivo. "En el modelo simultáneo el componente profesional se ofrece desde el inicio de la formación de manera simultánea a la formación general y/o al estudio de la(s) materia/s que el futuro docente pretende impartir". Este modelo es seguido por casi todos los países como el más idóneo para los niveles de la educación infantil y primaria. En países como Bélgica, Dinamarca, Alemania, Eslovaquia y Turquía, siguen este modelo en la formación de los profesores de enseñanza secundaria inferior (Eurydice, 2012a: 111). En cambio, en el modelo consecutivo "los estudiantes cursan primero sus estudios académicos de materia para, una vez finalizado su título académico, realizar el componente profesional en educación". Algunos países para la enseñanza secundaria ofrecen los dos modelos y otros en cambio para la enseñanza secundaria ofrecen solo el modelo consecutivo. En Alemania y Eslovaquia el modelo simultáneo es la única vía de acceso a la docencia en cualquier nivel educativo de la enseñanza, en cambio en Francia solo existe el modelo consecutivo (Eurydice, 2012a: 111).

b) Titulación de los docentes. En cuanto a las titulaciones requeridas para la incorporación de los docentes en los niveles de la enseñanza existen diferencias entre unos u otros países de la UE:

- Nivel de primaria. Respecto de los futuros profesores de primaria, la mayoría de los países europeos, España entre ellos, "exigen estar en posesión del título de Grado o equivalente de una duración de tres o cuatro años", sin embargo en otros países exigen un Máster con una duración de cinco años. 
- Nivel de secundaria. Respecto de la titulación para la docencia en la enseñanza secundaria los países se reparten en al 50\%: en una mitad basta la titulación de grado y en la otra mitad, España entre ellos, se exige la titulación de Grado y Máster (cinco años). En algunos países los profesores noveles deben realizar un "período de inducción adicional a su cualificación académica y profesional, en general, inmediatamente después de obtener su titulación. Este período suele consistir en un programa estructurado de apoyo a los futuros docentes" (Eurydice, 2012a: 112)

c) La formación inicial del profesorado en el ámbito español. Tomando como ejemplo el profesor de matemáticas, en el nivel de la enseñanza secundaria obligatoria y de bachillerato, la formación inicial viene configurada por el Grado en matemáticas y el Máster para enseñar matemáticas. El Grado tiene un diseño que integra contenidos, objetivos, competencias generales y transversales, normas y valores con los que se considera que el graduado moderno está técnicamente aprovisionado para su ejercicio profesional. Una segunda aportación converge a este desarrollo formativo: las competencias que el proceso de la enseñanza aprendizaje exige y la legislación vigente, nacional y europea, establece para la formación del profesor, tanto en la etapa de la graduación como en la siguiente del Máster (Tribó, 2008: 183). Una tercera aportación formativa, la necesidad de incorporar los recursos tecnológicos e informáticos tanto en la construcción del conocimiento como en su enseñanza viene establecida por la legislación vigente. La formación pedagógica del profesorado de la enseñanza secundaria obligatoria y del bachillerato se ha constituido en un proceso de formación reglado oficialmente a nivel de postgrado, con denominación de Máster, que se obtiene mediante sesenta créditos ECTS y son las universidades las encargadas de implementar este proceso educativo (ORDEN MINISTERIAL ECI/3858/ 2007/ de 27 de diciembre. BOE, núm.312, sábado 29 de diciembre 2007).

d) Aspectos complementarios sobre el profesorado. Estimo interesante mencionar algunas otras dimensiones vinculadas con el profesorado de la UE que coadyuvan a una idea más exacta sobre la formación y el desempeño de la docencia en la UE en los niveles de infantil, primaria y secundaria. 1) Siendo el profesor el eje axial de la enseñanza, para los centros educativos "resulta fundamental reclutar, formar y retener al personal docente cualificado", sin embargo según datos de los estudios PISA, un $15 \%$ de los alumnos de 15 años manifestaron carecer de personal cualificado para matemáticas y ciencias y en algunos países estos porcentajes se elevaron al 40\%. En España este asunto, de momento, no constituye un problema. 2) Las medidas de apoyo a los docentes noveles se están generalizando en los países de la UE para superar las dificultades inherentes a su reciente ingreso en la profesión. En otros casos se orientan a reducir la probabilidad de abandono profesional. En algunos países las medidas de apoyo tienen carácter general y aseguran la prestación del apoyo, en otros, en cambio, es potestativo de los centros proporcionarlas. 3) En cuanto a la relación contractual de los profesores las dife- 
rencias son múltiples: "En la mitad de los países estudiados la relación laboral de los profesores generalmente se establece mediante un contrato indefinido sujeto a la legislación laboral general. Como empleados del sector público, los docentes son contratados por la administración local, o por los centros, aunque suelen estar empleados directamente por el centro en el que desarrollan su labor." ( Eurydice, 2012a: 116)

\subsection{La formación metodológica del profesor}

La formación metodológica del profesor en el ámbito de la educación española está exigida y se contempla tanto en el diseño del Grado como del Máster, que constituyen los procedimientos de formación inicial del docente de la enseñanza secundaria y del bachillerato. El trabajo práctico con que concluyen ambos procesos se ha diseñado con el objetivo de formar, desde la perspectiva de la metodología didáctica, al futuro profesor: "Los enfoques pedagógicos y la metodología utilizada en los centros educativos para enseñar matemáticas pueden tener un gran impacto no solo sobre cuánto aprenden los alumnos en el aula, sino también sobre la calidad de dicho aprendizaje. Una metodología apropiada puede mejorar el nivel de comprensión de los alumnos. Los métodos didácticos también influyen en la forma en que los estudiantes se implican en su aprendizaje y en cómo lo disfrutan, lo que, a su vez, repercute directamente sobre la cantidad y la calidad de lo que aprenden" (Eurydice, 2012b: 57). Sin embargo cuando se trata de especificar un método concreto de aprendizaje las propuestas son variadas. La opinión más generalizada es que no existe un único método adecuado y los profesores han de seleccionar los métodos y estrategias acordes a la materia a explicar, al tipo de alumnos y al contexto del aprendizaje. Dentro de las actuales alternativas más comunes son el uso de las nuevas tecnologías en el aula y el aprendizaje cooperativo.

Sobre la influencia de las nuevas tecnologías en el aula existen estudios que son testigos del efecto positivo en la motivación y en el aprendizaje de los alumnos (Méndez, 2012a, 2012b). Los estudios internacionales más recientes indican que, en la práctica, se están manejando diferentes enfoques pedagógicos, en especial el aprendizaje cooperativo tiene efectos positivos en la motivación y en el aprendizaje (Méndez, 2012c, 2013).

\subsubsection{Cuestiones previas al aprendizaje cooperativo}

Se muestran dos aspectos interesantes: las formas de organizar la clase y una tabla comparativa entre el aprendizaje tradicional y cooperativo. En cuanto a la organización de la clase se pueden adoptar tres enfoques, el competitivo, el individualista y el cooperativo.

$1^{\circ}$ ) La estructura competitiva se apoya en unas determinadas creencias: la competición es inevitable, pues es connatural al ser humano; la competición es deseable pues estimula para alcanzar el éxito y competir es divertido y forma el carácter. Se podría matizar este planteamiento con variados argumentos. Baste 
señalar que los éxitos son escasos y en consecuencia la mayoría del alumnado acumularía experiencias de fracaso, con el grave contratiempo que ello supondría para su autoestima.

$2^{\circ}$ ) En la estructura individualista, el educando se esfuerza para alcanzar los objetivos sin influir en las metas de otras personas. Cada estudiante trabaja únicamente en beneficio propio y recibirá su recompensa de acuerdo a su trabajo personal con independencia de los resultados de los demás. En este enfoque no se cultivan las habilidades sociales.

$3^{\circ}$ ) En la estructura cooperativa, cada estudiante alcanza sus metas solo si las personas con las que trabaja alcanzan las suyas. Se hace responsable de su propio aprendizaje y del de los demás. Favorece el desarrollo de habilidades sociales y del trabajo en equipo (Gavilán, 2004).

\subsubsection{Variables del aprendizaje cooperativo}

En cuanto a las variables del grupo cooperativo, que se consideran relevantes, destacaría las relaciones intragrupales, el número de integrantes del grupo y su duración.

1) Relaciones. El grupo cooperativo se caracteriza por la responsabilidad que el estudiante ha de asumir sobre su propio aprendizaje y sobre el de los otros que integran el grupo, lo que no significa que la persona mejor preparada ejerza de profesor de los restantes miembros del grupo. Según la profesora Gavilán este tipo de grupos se caracteriza por:

- El objetivo es que todos los integrantes del grupo obtengan los mejores resultados en el aprendizaje.

- Cada miembro del grupo ha de asumir la responsabilidad del propio aprendizaje y del de los otros integrantes del grupo.

- En los grupos cooperativos el conocimiento se alcanza mediante las discusiones y la búsqueda de consenso, en cuyos procesos los integrantes se convierten en fuentes de conocimiento para sus compañeros.

- Para el funcionamiento del grupo es preciso que los integrantes posean habilidades sociales para resolver los conflictos de manera civilizada y coordinarse para hacer las tareas. El profesor no debiera dar por supuesto que los miembros del grupo poseen estas habilidades.

- Los resultados a alcanzar serán logros académicos y personales, como consecuencia de la confianza conseguida y de la interacción, que serán valoradas como superiores a lasque obtendrían mediante el trabajo individual.

2) Tamaño y duración del grupo. En relación con el tamaño del grupo, la profesora Gavilán considera que no deben ser numerosos, puesto que esta circunstancia 
podría emboscar personas que no aportaran al trabajo común y se beneficiaran en cambio de los esfuerzos ajenos. Esta situación sería evidente para los miembros del grupo, en cambio constituiría una notable dificultad identificarla desde fuera del grupo, que se vería progresivamente desmotivado en el trabajo, al observar que están haciendo el trabajo de otro que en nada colabora. Entre tres y cinco puede ser un número acertado de integrantes del grupo, siempre dependiendo del tipo de actividad de que se trate. Por lo que respecta a la duración del grupo, para la profesora Gavilán, los criterios desde los que valorar el asunto son la confianza y compromiso que ha de generarse en el grupo, mediante sus interacciones.El sentimiento de pertenencia al grupo requiere que se disponga del tiempo necesario para que se haga realidad (Gavilán, 2004).

\subsubsection{Modalidades del aprendizaje cooperativo}

Según Gavilán (2004), el método de trabajo cooperativo presenta dos características comunes: $1^{\mathrm{a}}$ ) el grupo de clase se subdivide en subgrupos y $2^{\mathrm{a}}$ ) entre los integrantes de cada subgrupo se crea una interdependencia positiva, mediante las tareas y/o sistemas de recompensa, que le animen a funcionar en equipo. Ha de aparecer a su consideración que en este tipo de aprendizaje se valora la importancia del proceso de aprendizaje y no solo el resultado final que pueda lograrse. A continuación resumimos algunas de las modalidades más importantes del aprendizaje cooperativo.

Learningtogether. Los hermanos David y Roger Johnson (1999) crearon un método cooperativo dando importancia a las habilidades sociales, que se consideran imprescindibles para el buen funcionamiento del procedimiento. El profesor organiza los grupos y los pasos necesarios para su correcto desarrollo son: 1) Seleccionar una lección bien estructurada.2) Decidir el tamaño del grupo y distribuir materiales. Dar instrucciones del trabajo y enumeración de objetivos. Explicar los criterios de éxito.3) El profesor observa, controla y supervisa el trabajo de los grupos. 4) Se evalúa el aprendizaje con una prueba personal y se calcula la puntuación del grupo que es la media de las puntuaciones de sus componentes.5) Puesta en común para analizar el funcionamiento de los grupos.

$J I G S A W$. Este método diseñado por Aroson en 1978, fue uno de los primeros que se crearon para implementar el aprendizaje cooperativo en el aula. Se caracteriza por establecer una fuerte interdependencia entre los miembros de cada equipo, de modo que el trabajo de cada estudiante es imprescindible para completar la tarea del grupo. Se organizan los grupos lo más heterogéneos posibles de entre 3 y 6 personas. El tema se descompone en tantaspartes como miembros de cada equipo hay y se realiza la entrega de una parte a cada componente del equipo que la completa, la aprende y se la prepara para enseñársela al resto del grupo. Los estudiantes de los diferentes grupos que están preparando la misma parte se reúnen para poner en común sus ideas y dificultades. Cuando vuelven a su grupo, tras haber asimilado su parte se la exponen a los compañeros de su equipo. Como la evaluación se hará a cada individuo de todo el tema, cada miembro tendrá que aprender lo que sus compañeros les han enseñado. 
Teams-Games-Tournament (TGT). Este método es una combinación de trabajo en grupo cooperativo (Teams), juegos instructivos (Games) y competición intergrupal (Tourmanent), creado por De Vries y Slavin(1976). Los pasos son los siguientes:1) El profesor comienza explicando la lección.2) Se organizan los grupos heterogéneos de cinco a seis personas. 3) Se introducen los juegos académicos con los que se trata de mejorar la actitud de los estudiantes hacia la tarea, salvando las diferencias individuales y mejorando las habilidades sociales. Los participantes representan a sus equipos en los juegos. 4) Se incorpora la competición entre los grupos para reforzar la motivación y la cohesión.Cada semana se modifican las competiciones para asegurar la interrelación y participación de todas las personas de la clase.

TeamAssistedIndividualization (TAI). Es un método ideado por Slavin (1985) para la enseñanza de las matemáticas cuando el grupo es muy heterogéneo. Los alumnos realizan una prueba inicial para determinar el nivel. Entonces se forman grupos lo más heterogéneos posibles de cuatro a cinco personas. Cada persona tiene que completar las hojas de actividades que se le entreguen que corresponden a su nivel, trabajan individualmente aunque pueden ayudarse unos a otros y aclarar dudas. Cada hoja contiene varios bloques de similar dificultad, de modo que cuando se supera el primer bloque correctamente se puede pasar a la siguiente hoja, en caso contrario, se iniciará el siguiente bloque de la misma hoja. Una vez completada la tarea, los estudiantes forman parejas elegidas por ellos mismos, con las que intercambian sus hojas de respuestas. Cada persona revisa la hoja de la pareja y la califica.

Peer Instruction. Método de aprendizaje cooperativo del que se da noticia en bastantes artículos de investigación (Bará, Domingo y Valero, 2007). El acto comunicativo se ha transformado de uno a varios emisores - profesor y alumnos- y varios receptores -alumnos y profesor. Se basa en que los alumnos se leen los contenidos previamente a la clase en la que se van a tratar, responden una serie de preguntas al empezar la clase y discuten sus respuestas con los compañeros de grupo; después se les proporcionan las mismas preguntas, que se volverán a contestar con alguna diferencia de las previas, debido a la discusión. En este punto confluyen otras versiones diferentes, como que el profesor explique si las respuestas siguen siendo erróneas. Esta metodología se realizó en Harvard y tiene un efecto en el aprendizaje notorio, además de que motiva a los estudiantes. Incluso en la actualidad se están implementando ciertas herramientas informáticas, de tal forma que, el profesor, al realizar los grupos, coloque a los alumnos que tienen diferentes respuestas en el mismo grupo, para facilitar así la discusión (Méndez y García-Alonso, 2013).

\subsubsection{El aprendizaje cooperativo en matemáticas}

Las matemáticas ocupan un lugar privilegiado en la aplicación de estos procedimientos que, a veces, surgieron precisamente como exigencias metodológicas para hacer frente a las cotas altas de fracaso escolar. Sobre la metodología de la enseñanza matemática se ocupó ampliamente el informe de la UE sobre el aprendizaje de las matemáticas y en él se señala que son variados los procedimientos según la diversidad de los estudiantes, el medio de pertenencia y la maduración de las capacidades 
del alumnado y algunos investigadores "señalan que métodos diferentes pueden funcionar bien en distintos contextos, mientras que otros afirman que los profesores deberían seleccionar el método más apropiado para su contexto educativo y para cada resultado del aprendizaje en concreto (...) En la mayoría de los países de la UE la administración central recomienda o establece una serie de métodos didácticos" (Eurydice, 2012b: 58).

Como resultado de las investigaciones llevadas a cabo en torno a la aplicación del procedimiento cooperativo a contenidos matemáticos de álgebra, cálculo y análisis, la profesora Gavilán (2004) extrae tres conclusiones interesantes:

$1^{\text {a) }}$ El aprendizaje matemático mejora con los procedimientos cooperativos cuando los estudiantes tienen la madurez apropiada para construir conceptos y relaciones que se establecen entre ellos, teniendo la oportunidad de interactuar con otros.

$2^{\mathrm{a}}$ ) En la enseñanza-aprendizaje de las matemáticas el intercambio intelectual desempeña un importante papel y los grupos cooperativos son un espacio ideal para que se produzcan.

$3^{\text {a }) ~ E l ~ a p r e n d i z a j e ~ c o o p e r a t i v o ~ c o n t r i b u y e ~ a ~ q u e ~ l o s ~ e s t u d i a n t e s ~ a u m e n t e n ~ l a ~}$ confianza en sus habilidades y adquieran autodisciplina, además de promover actitudes favorables hacia la asignatura.

\subsubsection{Decisiones y actividades del profesor en el aprendizaje cooperativo}

En la puesta en marcha de este procedimiento didáctico la actitud, decisiones y actividades del profesor constituyen un elemento esencial para que la nueva orientación metodológica consiga alcanzar la meta propuesta: la mejora de los resultados en el aprendizaje. Mencionamos únicamente algunos de los asuntos que ha de resolver, dirigir $u$ orientar el profesor:

1. Tamaño de los grupos y formación de los grupos.

2. Distribución en el aula y control de la efectividad.

3. Objetivos de cada lección y planteamiento de la tarea.

4. Intervenciones del profesor y evaluación del aprendizaje.

5. Revisión del funcionamiento de los grupos.

\section{Conclusiones}

De lo tratado con anterioridad en torno a los enfoques metodológicos, que preocupan a los profesores noveles y no tan noveles en las presentes circunstancias, se infiere lo que sigue: 
$1^{\circ}$ ) Ciertas coincidencias entre las investigaciones y las pruebas de conocimientos y competencias que suelen hacerse a nivel internacional por entidades solventes:

Es imposible afirmar que exista una única forma correcta de enseñar la materia de estudio, aun las matemáticas.

Diversos métodos pueden resultar eficaces cuando se aplican a contextos concretos y en función de los resultados del aprendizaje que se pretende alcanzar.

Es necesario encontrar un equilibrio entre la metodología que fomenta el aprendizaje del conocimiento matemático y la que potencia el desarrollo de habilidades y competencias en matemáticas y ciencias.

$2^{\circ}$ ) Teniendo en cuenta que el profesor juega el papel más importante, en cuanto a la implementación de las reformas educativas, sean estas sobre contenidos o sobre metodologías didácticas para la enseñanza de la materia, se han de tomar en consideración algunas precisiones:

Para ayudar a los estudiantes, los profesores han de ser capaces de escoger entre una amplia gama de métodos didácticos y ser capaces de motivar a todo tipo de alumnos y en particular, reforzar a aquellos que tienen bajos niveles del rendimiento.

Los programas de formación permanente del profesorado, promovidos a nivel central, abordan una serie de competencias para fomentar la incorporación de elementos innovadores en las prácticas docentes.

Las evaluaciones de los profesores y las oportunidades de formación permanente muestran la necesidad de un nuevo enfoque: fomentar en los profesores la adquisición de conocimientos matemáticos para la enseñanza.

$3^{\circ}$ ) En la mayor parte de los países que integran la UE se está promocionando, de manera creciente,"el área de formación permanente relacionada con la cooperación, la colaboración y el intercambio de experiencias entre profesores, particularmente a través de Internet con páginas web, blogs y redes sociales. Los resultados de los estudios confirman que es fundamental apoyar este tipo de comunidades virtuales, ya que animan a los profesores a aprender unos de otros y pueden contribuir a conseguir avances a mayor escala" (Eurydice, 2012b:160).

\section{Referencias bibliográficas}

BARÁ, J.; DOMINGO, J. y VALERO, M. (2007). Técnicas de aprendizaje cooperativo. Barcelona: Taller de formación en la Universidad Politécnica de Cataluña.

DE VRIES, D.; SLAVIN, R. (1976). Teams-Games-Tournament: A Final Report on Children Tutoring Children.Baltimore: Johns Hopkins University.

EURYDICE, (2012a). Cifras Clave de la Educación en Europa 2012.Madrid: Secretaría General Técnica del MEC y D. 
(2012b). La enseñanza de las matemáticas en Europa: retos comunes y politicas nacionales. Madrid: Secretaría General Técnica del MEC y D.

GAVILÁN, P. (2004):Álgebra en secundaria. Trabajo cooperativo en matemáticas. Madrid: MEC y Narcea.

JOHNSON, D.; JOHNSON, R. (1999).Learning together and alone.Cooperative, competitive and individualistic learning.Engllewood Cliffs. Prendice-Hall, Inc. New Jersey.

MÉNDEZ, D. (2012a). "Cambio motivacional realizado por las TIC en los alumnos de secundaria de Física".En: Miscelánea de Comillas, no 70 (136) p. 199-224.

(2012b)."The experience of learning physics through the application of ICT". En:Energy Education Science and Technology Part B. Social and Educational Studies, no4(1), p. 674-679.

(2012c)."El aprendizaje cooperativo y la enseñanza tradicional en el aprendizaje de la física". En: Educación y futuro, no 27, p. 179-200.

(2013)."The influence of teaching methodologies in the learning of thermodynamics in secondary education". En:Journal of Baltic Science Education, no 12 (1), p. 59-72.

MÉNDEZ, D.; GARCÍA-ALONSO, P. (2013). "Cambio comunicativo en las nuevas metodologías didácticas". En:Estudios sobre el mensaje periodístico, ${ }^{\circ} 19$, p. 299-308. Disponible: http://dx.doi.org/10.5209/rev_ESMP.2013.v19.42037[1010-2013]

ORDEN MINISTERIAL ECI/3858/ 2007/ de 27 de diciembre.

SLAVIN, R. E., (2009). "What Works in teching maths?", en Better: evidence-based Education, 2,1, 4-5 (Online). http://content.yudu.com/A1i1c9/BetterFallo9US (Consultado el 8 de noviembre de 2012).

TRIBÓ, G. (2008). "El nuevo perfil de los profesores de secundaria". En:Educación $X X I, \mathrm{n}^{\circ} 11$, p. 183-209.

VASSILIOU, A.; SEMETA, A., (2012). Prólogo.En Eurydice, Cifras Clave de la Educación en Europa 2012. Secretaría General Técnica del MEC y D. Madrid.

\section{Los autores}

Miriam Méndez Coca. Nacida el 2/2/1975 en Madrid (ESPAÑA). Licenciada en Matemáticas en Universidad Complutense de Madrid y doctora en Pedagogía en Universidad Pontificia de Salamanca con la tesis "Enseñanza-aprendizaje de las matemáticas en la E.S.O." defendida el 2013 obteniendo una calificación de Cum Laude. Trabajé como analista de programación para Unión Fenosa y BBVA 2000 2009. Máster en Ingeniería de Software 2002-2004. Profesora de secundaria y bachillerato desde 2010 en diferentes centros públicos, privados y concertados. Profesora del centro universitario de Villanueva (UCM) desde el 2013. Título de inglés de la Escuela Oficial de Idiomas. 
David Méndez Coca. Licenciado en Ciencias Físicas en la UCM (2002) y doctor en Educación en la UCM (2011). Premio extraordinario de doctorado. Profesor de secundaria y bachillerato durante diez años. Autor de más de diez artículos publicados en revistas españolas y extranjeras, más de quince comunicaciones en congresos en México, Estados Unidos, República Checa, España, etc. Profesor en el Centro Universitario Villanueva desde el 2011. 